\title{
Effects of homogenous soil characteristics to the transient responses of a single long horizontal ground conductor model
}

\begin{abstract}
This study presents various transient responses behaviours of a single long horizontal ground conductor due to various types of its surrounding homogenous soil. The study is done in time domain using TLM method for the transient node voltages and transient node currents along the ground conductor are demonstrated to be dissipated faster into its surrounding soil with a low resistivity value. It also shown that soil at low hills with rich soil and trees environments is more suitable to be the burial medium of a ground conductor compared to soil at high-rise city streets and industrial areas.
\end{abstract}

Keyword: Homogeneous soil; Single long horizontal ground conductor; Soil permittivity; Soil resistivity; TLM; Transient responses 